\title{
Risk factors associated with vancomycin-resistant enterococcus (VRE) and methicillin-resistant Staphylococcus aureus (MRSA) co-infection
} Zervos $^{1,2}$, and Katherine Reyes ${ }^{1 *}$

${ }^{1}$ Henry Ford Hospital, Detroit, MI, USA

${ }^{2}$ Wayne State University, School of Medicine, Detroit, MI, USA

\begin{abstract}
A retrospective case control study evaluated risk factors for co-infection with methicillin resistant Staphylococcus aureus (MRSA) and vancomycin-resistant enterococci (VRE). Over an 8-year period, 111 patients with MRSA and VRE were identified as cases and 1077 patients with MRSA alone were controls. The variables collected were age, gender, previous antibiotic administration, bacterium strain type and underlying comorbidities. Independent risk factors of co-infection were exposure to vancomycin $(\mathrm{OR}=3.70)$, quinolones $(\mathrm{OR}=3.09)$ and cephalosporins $(\mathrm{OR}=2.03)$ up to 3 months before bacterial isolation, neurologic disease $(\mathrm{OR}=2.22)$, gastrointestinal disease $(\mathrm{OR}=1.95)$, respiratory disease $(\mathrm{OR}=2.00)$ renal disease $(\mathrm{OR}=1.67)$, diabetes $(\mathrm{OR}=1.83)$ and dialysis $(\mathrm{OR}=1.92)$. The results of the study will have important implications for control interventions.
\end{abstract}

\section{Introduction}

Antibiotic resistance is a growing public health concern among researchers and health care professionals worldwide. The ability of a pathogen to effectively resist antibiotics displays the complexity of these organisms as well as the difficulties they present when treating patients. Nosocomial MRSA infections have spread beyond hospitals, infecting patients in health care settings such as nursing homes and rehabilitation facilities. Both MRSA and VRE have been identified as the most common antimicrobial-resistant pathogens associated with health care-associated infections [1]. Community acquired MRSA is also becoming more common, infecting previously healthy individuals who have not had contact with healthcare facilities [2].

According to the World Health Organization (WHO), 5 out of 6 WHO regions have reported $50 \%$ or greater resistance of Staphylococcus aureus to methicillin [3]. Vancomycin, the therapy of choice for MRSA, became increasingly used since the 1970 's but by 1988 , there were cases of vancomycin resistant enterococcus (VRE) being reported [4]. Enterococcus faecium and Enterococcus faecalis are the two most common species responsible for enterococcal infections in humans [5]. E. faecium and E. faecalis are also two distinct organisms in that their VRE strain type identification has different implications. If these strains spread further, they can have devastating consequences

The objective of this study is to identify risk factors associated with antibiotic resistance in patients co-infected with $S$. aureus and enterococcus. As of 2012, Vancomycin-resistant S. aureus (VRSA) has been isolated from 14 patients across the United States, the first and eight of which were from southeast Michigan [6]. All cases of VRSA in the U.S. began as MRSA and acquired vancomycin-resistance through co-colonization between MRSA and VRE. While it is unclear exactly why cases are more prevalent in this region compared to the rest of the country, previous research studies have discovered that VRE strains can transfer the vanA gene complex to $S$. aureus, leading to VRSA [5]. VRE strains containing a particular plasmid that facilitates the transfer of the VanA gene to $S$. aureus have been found to be more prevalent in Michigan [5]. The first case of vanA-mediated VRSA was identified in 2002 from a foot wound in a patient with diabetes who was receiving long-term vancomycin therapy [7].

\section{Methods}

The study design is a retrospective case-control, with patients who have co-infection of MRSA and VRE acting as the cases and patients with MRSA only acting as the controls. The study period is from 2006-2014 and included patients at Henry Ford Health System (HFHS) who were identified to have co-infection of MRSA as well as either E. faecium or E. faecalis. Study subjects were identified via review of microbiology records. HFHS is an 800-bed teaching hospital located in urban Detroit. MRSA strains were isolated from blood while enterococcus was isolated from various sources including blood, urine, wounds, peritoneal fluid, and biopsy tissue.

Individual patient information collected included year and age at the time of first infection, gender, number of days between MRSA and VRE isolates (for cases), MRSA and VRE strain type, previous antibiotic administration within three months of first infection, and co-

Correspondence to: Katherine Reyes, Henry Ford Health System, Division of Infectious Diseases, 2799 West Grand Blvd CFP3, Henry Ford Health System, Detroit, MI, 48202, USA, Tel: 313-916-2573; E-mail: kreyes1@hfhs.org

Key words: Staphylococcus aureus, methicillin-resistant, vancomycin-resistant Received: July 06, 2016; Accepted: July 28, 2016; Published: August 02, 2016 
morbidities. This information was extracted from electronic medical records stored on the HFHS server.

Identification of isolates and in vitro susceptibility was done by the clinical microbiology laboratory using Clinical and Laboratory Standards Institute (CLSI) standards.

Pulsed-field gel electrophoresis (PFGE) of Sma1 digested genomic DNA was performed using CHEF-DRIII (Bio-rad). PFGE patterns were compared using Bionumerics software (Applied Maths). Isolates were considered in the same group if greater or equal to 80 percent similar by dice coefficient

Statistical analysis was performed using Chi-square and Fischer's exact tests to determine statistical significance. A P-value less than .05 was determined to be statistically significant and these results are bolded. Stepwise selection was used to determine independent risk factors for inclusion in the final model. All analyses were conducted using Stata version 14.0.

\section{Results}

In total, 1077 patients were identified to have MRSA bacteremia and 111 had both MRSA bacteremia and VRE. Forty seven percent of cases were male and the mean age was 59.9 with a range of 20-89. Sixty percent of controls were male and the mean age was 60.5 with a range of 18-101. Univariate analysis (Table 1) revealed a statistically significant difference in previous antibiotic use among cases and controls for vancomycin, betalactam, cephalosporin, carbapenem, macrolide, clindamycin, quinolone and aminoglycoside. There was a statistically significant difference in cardiac disease, diabetes, neurologic disease, kidney disease, respiratory disease, and dialysis between cases and controls. USA 100 was the most abundant MRSA strain among cases (52.3\%) while USA 300 was more common among controls (44\%).

Of the 111 MRSA + VRE patients, 61 isolates were identified as $E$. faecium and 52 isolates were identified as E. faecalis. Strain 1 was the most abundant type in both faecium and faecalis with $16.4 \%$ and $64.5 \%$ respectively (Table 2 ).

From the multivariate analysis (Table 3), independent risk factors for co-infection of MRSA and VRE were identified to be previous exposure to vancomycin, quinolones and cephalosporins three months prior to isolation of bacterium, history of neurologic disease, respiratory disease, GI disease, diabetes mellitus, renal disease and dialysis treatment.

\section{Discussion}

Previous studies have focused on risk factors for MRSA or VRE alone and found that patients with VR E. faecalis were more frequently exposed to cephalosporins than patients with VR E. faecium [5]. Studies have also found fluoroquinolones to be associated with isolation of VR E. faecalis [5]. Reyes et al. [1] found isolation of VR E. faecalis and prior use of linezolid or clindamycin to be independent risk factors for cocolonization or co-infection with VRE and MRSA [1]. There are very limited earlier data on comparison between MRSA and VRE dual infection and MRSA alone. Our study is unique in that it defined VRE to include both enterococcal organisms and found cephalosporin and quinolone exposure to be independent risk factors for co-infection of MRSA and VRE.

The reason VRSA was first identified in Detroit, and 8 of the first patients with VRSA were from Southeastern Michigan remains a mystery. There is a hypothesis that there are unique patient or
Table 1. Univariate Analysis of risk factors for co-infection of MRSA and VRE, Henry Ford Hospital 2006-2014

\begin{tabular}{|c|c|c|c|}
\hline & MRSA & MRSA + VRE & P-value \\
\hline Total \# of patients & 1077 & 111 & \\
\hline Males: Females & $644: 436$ & $52: 60$ & 0.005 \\
\hline Mean age (range) & $60.5(18-101)$ & $59.9(20-89)$ & 0.955 \\
\hline \multicolumn{4}{|l|}{ Previous ABX use } \\
\hline Vancomycin & $194(18)$ & $65(58.6)$ & $<.0001$ \\
\hline Linezolid & $30(2.8)$ & $8(7.2)$ & 0.012 \\
\hline Daptomycin & $10(0.9)$ & 0 & 0.612 \\
\hline Trimsulfa & $26(2.4)$ & $4(3.6)$ & 0.517 \\
\hline Tetracyclines & $7(0.6)$ & $3(2.7)$ & 0.059 \\
\hline Penicillins & $31(2.9)$ & $4(3.6)$ & 0.561 \\
\hline Betalactam & $46(4.3)$ & $21(18.9)$ & $<.0001$ \\
\hline Cephalosporins & $157(15)$ & $56(50.5)$ & $<.0001$ \\
\hline Carbapenem & $17(1.6)$ & $13(11.7)$ & $<.0001$ \\
\hline Metronidazole & $59(5.5)$ & $26(23.4)$ & $<.0001$ \\
\hline Macrolides & $32(3)$ & $9(8.1)$ & 0.005 \\
\hline Clindamycin & $19(1.8)$ & $10(9)$ & $<.0001$ \\
\hline Quinolones & $85(7.9)$ & $37(33.3)$ & $<.0001$ \\
\hline Aminoglycosides & $39(3.6)$ & $19(17.1)$ & $<.0001$ \\
\hline other & $46(4.3)$ & $8(7.2)$ & 0.157 \\
\hline \multicolumn{4}{|l|}{ Comorbidities } \\
\hline Cancer & $118(11)$ & $17(15.3)$ & 0.168 \\
\hline Cardiac & $292(27)$ & $47(42.3)$ & 0.001 \\
\hline Diabetes & $420(39)$ & $65(58.6)$ & $<.0001$ \\
\hline GI disease & $217(20)$ & $30(27)$ & 0.08 \\
\hline HIV & $32(3)$ & $3(2.7)$ & 1.00 \\
\hline Neurologic & $212(20)$ & $42(37.8)$ & $<.0001$ \\
\hline PVD & $135(13)$ & $20(18)$ & 0.102 \\
\hline Kidney Disease & $496(46)$ & $75(67.6)$ & $<.0001$ \\
\hline Respiratory & $194(18)$ & $33(29.7)$ & 0.003 \\
\hline Dialysis & $221(21)$ & $40(36)$ & $<.0001$ \\
\hline \multicolumn{4}{|l|}{ Strain Type- MRSA } \\
\hline USA 100 & $458(43)$ & $58(52.3)$ & 0.049 \\
\hline USA 300 & $472(44)$ & $32(28.8)$ & 0.002 \\
\hline USA 400 & $5(0.5)$ & 0 & 1.00 \\
\hline USA 500 & $6(0.6)$ & 0 & 1.00 \\
\hline USA 600 & $16(1.5)$ & $5(4.5)$ & 0.039 \\
\hline USA 700 & $4(0.4)$ & 0 & 1.00 \\
\hline USA 900 & 0 & 0 & \\
\hline USA 1100 & $1(0.1)$ & 0 & 1.00 \\
\hline not $100-1100$ & $115(10.7)$ & $16(14.4)$ & 0.231 \\
\hline
\end{tabular}

${ }^{a}$ Data include percentages of patients. Statistically significant data are shown in bold. Abbreviations: GI, gastrointestinal; PVD, peripheral vascular disease

strain characteristics that account for this finding [8,9]. As of 2011, the prevalence of diabetes in Michigan is $16.7 \%$ compared to $8.3 \%$ prevalence in the U.S. as a whole [5]. Importantly, the frequency of diabetes in our study was high among cases (58.6\%) as well as controls (39\%) and was found to be a risk factor of co-infection. Patients with diabetes are at a higher risk for chronic skin and bone infections as they are more prone to skin ulceration and contact with the health care system, which can lead to co-colonization of the wounds with these organisms. Tosh et al. [8] looked at the prevalence and risk factors associated with VRSA precursor organisms in Southeastern Michigan in patients with chronic lower-extremity wounds. The study found that having long-standing chronic wounds was a risk factor for pSK41positive S. aureus colonization [8].

Hemodialysis and peritoneal dialysis are used to treat both acute and chronic renal disease by filtering a patient's blood through a permanent indwelling device. Past research indicates that indwelling 
Table 2. Strain type counts for VR E. faecium and VR E. faecalis, Henry Ford Hospital 2006-2014.

\begin{tabular}{|c|c|c|}
\hline Strain type & VR E. faecium & VR E. faecalis \\
\hline 1 & 10 & 33 \\
\hline 2 & 6 & 3 \\
\hline 3 & 5 & 3 \\
\hline 4 & 4 & 2 \\
\hline 5 & 4 & 1 \\
\hline 6 & 4 & 1 \\
\hline 7 & 2 & 1 \\
\hline 8 & 2 & 1 \\
\hline 9 & 2 & 1 \\
\hline 10 & 2 & 1 \\
\hline 11 & 2 & 1 \\
\hline 12 & 2 & 1 \\
\hline 13 & 2 & 1 \\
\hline 14 & 1 & 1 \\
\hline 15 & 1 & 1 \\
\hline 16 & 1 & \\
\hline 17 & 1 & \\
\hline 18 & 1 & \\
\hline 19 & 1 & \\
\hline 20 & 1 & \\
\hline 21 & 1 & \\
\hline 22 & 1 & \\
\hline 23 & 1 & \\
\hline 24 & 1 & \\
\hline 25 & 1 & \\
\hline 26 & 1 & \\
\hline 27 & 1 & \\
\hline
\end{tabular}

Table 3. Multivariate analysis of risk factors for co-infection of MRSA and VRE, Henry Ford Hospital 2006-2014.

\begin{tabular}{|l|c|c|}
\hline Variable & OR (95\% C.I.) & p-value \\
\hline Vancomycin & $3.70(2.20-623)$ & $<.0001$ \\
\hline Quinolones & $3.09(1.80-5.30)$ & $<.0001$ \\
\hline Neurologic disease & $2.22(1.38-3.56)$ & 0.001 \\
\hline Cephalosporins & $2.03(1.17-3.53)$ & 0.012 \\
\hline Respiratory disease & $2.00(1.21-3.31)$ & 0.007 \\
\hline GI disease & $1.95(1.17-3.27)$ & 0.011 \\
\hline Dialysis & $1.92(1.10-3.36)$ & 0.022 \\
\hline Diabetes & $1.83(1.15-2.91)$ & 0.01 \\
\hline Renal disease & $1.67(.97-2.85)$ & 0.063 \\
\hline
\end{tabular}

devices are independent predictors of VR E. faecalis isolation, and our study identified dialysis and renal disease to be risk factors of coinfection [5]. Renal failure patients are often treated with long durations of antibiotics. Respiratory disease and gastrointestinal disease were also identified as risk factors for co-infection although these variables have not been identified as risk factors by previous studies. Importantly, there was no specific strain type of MRSA associated with dual infection. Earlier studies have shown USA100 to be the predominant strain type associated with VRSA [9]. We found $54 \%$ of patients studied had VR E. faecalis vs. VR E. faecium. Prevalence of VR E. faecium generally ranges from 0 to a few percent in earlier studies. The significance of this observation is that VR E. faecalis is the most important precursor for VRSA in earlier studies.

This study has several limitations. For case patients, the time between isolation of MRSA and VRE varied anywhere from 0 days to 1755 days, which potentially affects the generalizability of the results. However, it is known that colonization with these organisms is generally felt to be long term (months to years) when it develops; therefore it is difficult to determine a specific timeframe that would imply an association. Further studies should be conducted analyzing risk factors among cases of co-infection within a specified time period. A second limitation of this study is its retrospective study design, and single center analysis in one geographic area. A third limitation is that only patients with MRSA bacteremia were included in this study. While information on the underlying source of infection was unavailable, patients would have been colonized with $S$. aureus bacteremia at multiple locations throughout the body.

The results of this study indicate an urgent need for reevaluation of antibiotic administration to patients. It also indicates the need to focus on patients with serious underlying disease for control interventions that might include different approaches to interrupt acquisition of the organism. Previous antibiotic exposure plays a large role in development of resistance and must be properly addressed in health care settings. Further study of where infections were likely acquired (community, health care setting) can also provide greater insight into optimal control interventions.

\section{References}

1. Reyes K, Malik R, Moore C, Donabedian S, Perri M, et al. (2010) Evaluation of risk factors for coinfection or cocolonization with vancomycin-resistant enterococcus and methicillin-resistant Staphylococcus aureus. J Clin Microbiol 48: 628-630. [crossref]

2. Ippolito G, Leone S, Lauria FN, Nicastri E, Wenzel RP (2010) Methicillin-resistant Staphylococcus aureus: the superbug. Int J Inf Dis 14: 7-11.

3. World Health Organization (2014) Antimicrobial resistance global report on surveillance. Geneva, Switzerland: WHO Press.

4. Hede K (2014) Antibiotic resistance: An infectious arms race. Nature 509: S2-3 [Crossref]

5. Hayakawa K, Marchaim D, Palla M, Gudur UM, Pulluru H, et al. (2013) Epidemiology of vancomycin-resistant Enterococcus faecalis: A case-control study. Antimicrob Agents Chemother 57: 49-55.

6. Albrecht VS, Zervos MJ, Kaye KS, Tosh PK, Arshad S, et al. (2014) Prevalence of risk factors for vancomycin-resistant Staphylococcus aureus precursor organisms in southeastern michigan. Infect Control Hosp Epidemiol 35: 1531-1534. [Crossref]

7. Limbago BM, Kallen AJ, Zhu W, Eggers P, McDougal LK, et al. (2014) Report of the 13 th vancomycin-resistant Staphylococcus aureus isolate from the United States. J Clin Microbiol 52: 998-1002.

8. Tosh PK, Agolory S, Strong BL, Verlee K, Finks J, et al. (2013) Prevalence and risk factors associated with vancomycin-resistant Staphylococcus aureus (VRSA) precursor organism Colonization among patients with chronic lower extremity wounds in Southeastern Michigan. Infect Control Hosp Epidemiol 34: 954-960. [Crossref]

9. Zhu W, Murray PR, Huskins WC, Jernigan JA, McDonald LC, et al. (2010) Dissemination of an Enterococcus Inc18-Like vanA Plasmid, Associated with Vancomycin-Resistant Staphylococcus aureus. Antimicrob Agents Chemother 54: 4314-4320. [Crossref]

Copyright: (C2016 Misikir HM. This is an open-access article distributed under the terms of the Creative Commons Attribution License, which permits unrestricted use, distribution, and reproduction in any medium, provided the original author and source are credited. 